\title{
Reply to the comment by Carmelo Anile on the paper (CNS-10-0256) "Complexity analysis of the cerebrospinal fluid pulse waveform during infusion studies"
}

Dear Editor,

We wish to thank Dr. Anile for his thoughtful comments shedding light on some shortcomings in our article featured in the December 2010 issue of Child's Nervous System (1). After having read the comments, the authors thought it would be convenient to clarify some of the concepts dealt with in the paper.

The scale of today's biomedical problems demands that clinical research physicians move beyond the confines of their individual disciplines. Studies dealing with the intracranial pressure (ICP) signal mostly progress based upon concepts and methods for which biomedical engineering background is advisable. This feature demands a broadening of research methods and a multidisciplinary approach to tackle these problems jointly. However, biomedical engineering is hardly an interdisciplinary field of study but a combination of postgraduate courses, which make a computer scientist or a mechanical or electrical engineer a biomedical one. This is -by experience- not enough because the nature of biomedicine is completely different; things are far removed from engineering theory, laboratory and technical standards. Hence, the development of these groups must overcome barriers of communication and understanding, which are inherent to each domain.

The time-averaged mean derived from the ICP signal was the first brain parameter to be monitored for clinical purposes. Qualitative analysis of the time-series generated by this signal still strives to help clinicians in the diagnosis and treatment of different pathologies. Usually, trained clinicians analyse them by visual inspection of the recording, a complex and time-consuming task that is also relatively subjective. Furthermore, a mere visual inspection might result in several characteristics of the signal not being detected. Therefore, the development of tools to facilitate its interpretation saving time, gaining objectivity and allowing further research is desirable.

Non-linearity is present in all physiological signals. They display irregular variations which at the same time are not random. A major feature of non-linear phenomena is the sensitivity of the generating system to variations in the initial conditions. Non-linear dynamics studies this feature as one cause of complex behaviour within the system. Physiological variability in ICP dynamics has indeed been acknowledged since the dawn of ICP monitoring. Thorough analysis of the pulsatile ICP signal through the visual inspection of printed recordings allowed recognition of the spontaneous variations in all components of the wave, as well as the changes in the wave configuration under specific conditions. This essentially qualitative analysis of the ICP trace soon led to the assumption that the single-wave morphology could have potentially important clinical implications. This expectation has gained relevance with a renewed interest in the pulsatile component of ICP dynamics $(2,3)$. We respectfully consider that the quoted sentence by Hirai et al. in 1984 (4) cited by Carmelo Anile (5) " ...the change in the waveform from polyphasic to monotonous..." should be regarded in this context, but should in no way be compared with the observation of a decreased complexity with the Lempel Ziv metric (1). Studies dealing with the ICP trace throughout the eighties relied largely on visual inspection systematizing fairly consistent wave components, therefore, using a reductionist approach. Moreover, this inability to measure the physiological variability may well have undermined the clinical significance of the ICP waveform.

Complex systems analysis provides tools to address this physiological variability. Clinicians who attempt to evaluate the relevance of these findings confront a confusing array of terms and concepts. Complexity is 
one of them. There is no single definition for this term, as it acquires different meanings in different contexts. Tononi et al. (6) shed some light with the following complexity measures descriptions:

- Dimensional complexity or just complexity: the non-linear method called correlation dimension.

- Kolmogorov or algorithmic complexity: according to Kolmogorov, the complexity of a given string of zeros and ones is given by the number of bits of the shortest computer program which can generate it.

- Neural complexity, defined in terms of integration or in terms of mutual information: statistical measures that capture regularities based on the deviation from independence among subsets of a system.

The Lempel-Ziv metric is an appropriate measure of complexity in Kolmogorov's sense that overcomes some of the disadvantages usually found in algorithmic complexity estimation, like computer dependency. The Lempel Ziv complexity is a nonparametric, simple-to-calculate measure of complexity in a onedimensional signal that does not require long data segments to compute. It is related to the number of distinct substrings and the rate of their recurrence along the given sequence, with larger values corresponding to more complexity in the data. For these reasons, we decided to characterise the ICP signal with this metric, performing a detailed inspection of its changes over time with a sliding window algorithm. In this way, we could examine the subtle complexity changes in the wave morphology. The Lempel-Ziv complexity analysis focuses on the pulsatile component of the pressure signal and it reflects the rate of new pattern occurrences in the investigated sequence.

The Lempel Ziv complexity is one of several non-linear analysis techniques which allows assessment of ICP dynamics in ways that are not possible with conventional methods. These strategies have been developed in the last decades since data acquisition systems with massive storage capacity are becoming more widespread. We believe that non-linear analysis methods - such as the Lempel-Ziv complexity could be powerful tools to understand ICP dynamics in ways which are not possible yet.

\section{REFERENCES}

1. Santamarta D, Hornero R, Abásolo D, Martínez-Madrigal M, Fernández J, García-Cosamalón J (2010) Complexity analysis of the cerebrospinal fluid pulse waveform during infusion studies. Child's Nerv Syst 26:1683-1689

2. Anile C, De Bonis P, Albanese A, Di Chirico A, Mangiola A, Petrella G, Santini P (2010) Selection of patients with idiopathic normal-pressure hydrocephalus for shunt placement: a single-institution experience. J Neurosurg 113: 64-73

3. Wagshul M, Eide P, Madsen J (2011) The pulsating brain: A review of experimental and clinical studies of intracranial pulsatility. Fluids and Barriers of the CNS 8:5 (doi:10.1186/2045-8118-8-5)

4. Hirai O, Handa H, Ishikawa M, Kim S-H (1984) Epidural pulse waveform as an indicator of intracranial pressure dynamics. Surg Neurol 21: 67-74

5. Anile C (2010) Comment to the article entitled "Complexity analysis of the cerebrospinal fluid pulse waveform during infusion studies" CNS-10-0256. Child's Nerv Syst 26:1691-1692

6. Tononi G, Edelman GM, Sporns O (1998). Complexity and coherency: integrating information in the brain. Trends Cogn Sci 2(12):474-484. 